\title{
RECURRENT EMERGENCY DEPARTMENT PRESENTATION - A CASE OF HEPATIC HYDROTHORAX
}

\begin{tabular}{ll}
$\begin{array}{l}\text { Mohammad Ismail } \\
\text { Nizami }\end{array}$ & $\begin{array}{l}\text { Assistant Professor, Department of Emergency Medicine ,Nizam's Institute } \\
\text { of Medical Sciences ,Hyderabad,Telangana State-500082,India. }\end{array}$ \\
\hline Vishwa Reddy & $\begin{array}{l}\text { Assistant Professor-(Ex), Department of Emergency Medicine, Nizam's } \\
\text { Institute of Medical Sciences ,Hyderabad,Telangana State -500082,India. } \\
\text { Gankidi* }\end{array}$ \\
\hline
\end{tabular}

ABSTRACT Introduction : In patients with advanced liver disease, dysregulation of extracellular fluid volume results in abnormal accumulation of fluid such as ascites, pleural effusion and edema .Hepatic hydrothorax occurs with accumulation of fluid $(\geq 500 \mathrm{ml})$ in patients with cirrhosis of liver in the absence of cardiopulmonary disease.The effusion which is transudate most commonly occurs in the right hemithorax .Treatment includes pharmacological-diuretics administration, therapeutic thoracocentesis, operative interventions like TIPSS would be required in relapsed and refractory cases. We present a rare case of a male patient, who presented to emergency with progressively worsening dyspnoea, having massive pleural effusion, was later diagnosed to have hepatic hydrothorax with cirrhosis of liver with portal hypertension, was stabilized and treated in the emergency department .

KEYWORDS : Inter costal drainage- ICD ;Hepatic hydrothorax in the absence of Ascites -HHAA ;Trans jugular intra hepatic portosystemic shunt-TIPSS; Hepatic Encephalopathy- HE

Background : Hydrothorax is an uncommon entity encountered in the emergency department. Hepatic hydrothorax is defined as significant pleural effusion (usually greater than $500 \mathrm{~mL}$ ) in a cirrhotic patient, in the absence of underlying pulmonary or cardiac disease. The incidence is found to be $5-10 \%$ in patients with decompensated cirrhosis ${ }^{(3)}$. The presentation is usually with dyspnoea, chest pain and abdominal distension. Pleural effusion is a common finding and it develops as a complication of cirrhosis of liver with ascites, portal hypertension as coexisting conditions complicated by hepatic encephalopathy or acute kidney injury ${ }^{(2)}$.

Case details : A 60 year old male presented to emergency with breathlessness and abdominal distension with h/o hypertension, diabetes mellitus and similar complaints in the past. Physical examination : Pulse-130 beats/min, BP -130/80 $\mathrm{mmHg}$,GCS -15/15 , Spo2 - 90\% ,Temperature -afebrile ;obese, conscious, coherent with mild pallor, icterus and pedal edema (pitting type). Systemic examination : Respiratory system : decreased breath sounds, dullness to percussion on the right side and basal crepitations were present, Cardiovascular system : Heart sounds regular, no murmurs ,Gastrointestinal system: Âbdomen was soft, distended and bowel sounds were present, Central Nervous system : Normal. Investigations : $\mathrm{ABG}$ - $\mathrm{Ph}-7.28, \mathrm{PCO}_{2}-56, \mathrm{HcO}_{3}-12, \mathrm{Po}_{2}-60$, Urea$144 \mathrm{mg} / \mathrm{dl}$, Creatinine $-2.2 \mathrm{mg} / \mathrm{dl}$, Hemoglobin- $7 \mathrm{mg} / \mathrm{dl}$, White blood cell count $-18000 / \mathrm{mm}^{3}$ Platelet count $-150000 / \mathrm{mm}^{3}$. Na+ $-128, \mathrm{~K}+-4.5, \mathrm{Cl}-96$. Total Bilirubin $-6 \mathrm{mg} / \mathrm{dl}$, Direct bilirubin$2.5 \mathrm{mg} / \mathrm{dl}$, Albumin $-2.3 \mathrm{mg} / \mathrm{dl}$, PT INR-1.08: Chest X ray Massive right sided pleural effusion (Figure 1), 2D EchoNormal , USG abdomen : Cirrhosis of liver with portal hypertension, ascites, oesophageal varices grade- IIIÎ and right sided effusion . Hepatic Hydrothorax was diagnosed as per the history and work up, which was suggestive of recurrent transudative type of pleural effusion(as per the Light's Criteria $)^{(5)}$ without any systemic pathology.

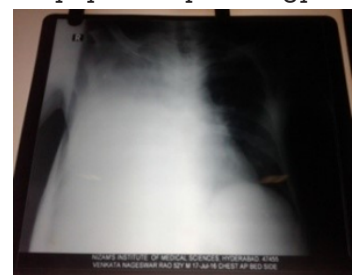

Fig: 1 Chest X ray S/o Massive right sided effusionHepatic Hydrothorax
Management :

Patient was stabilized in the ED with oxygen ,diuretics, analgesics and antibiotics. Terlipressin infusion was started ,diagnostic and therapeutic thoracocentesis was done which was suggestive of transudative type of effusion and intercostal drain insertion was planned. In view of respiratory failure patient was intubated, mechanical ventilatory support and peritoneal dialysis was initiated for the treatment of renal failure , computed tomography of the chest was ordered , later patient was admitted and transferred to respective department . Hepatic Hydrothorax in the absence of ascites (HHAA) is an uncommon presentation ${ }^{(4)}$. The pathophysiology of hepatic hydrothorax is not well defined, the most likely explanation is pressure gradient-directed unidirectional flow of ascites which occurs through a small diaphragmatic $\operatorname{defect}(\leq 1 \mathrm{~cm})$ into the pleural cavity, most commonly (85\%) right sided , (13\%) left sided,

( $2 \%$ ) occur bilaterally ${ }^{(1)}$.

\section{CONCLUSION}

Hepatic hydrothorax is one of the rare conditions to be identified by the emergency physician in patients presenting with recurrent pleural effusion and coexisting liver pathology . Management involves treatment of underlying chronic liver disease along with hepatic hydrothorax .It includes screening for the liver under the guidance of gastroenterologist or hepatologist, ascites management with aldosterone antagonists and loop diuretics, therapeutic thoracocentesis for symptomatic effusions in combination with low salt and high protein diet .Chest drainage and pleurodesis can be done for recurrent presentations with hydrothorax . Patients with hepatic hydrothorax usually have advanced liver disease and most of them require liver transplantation, so assessment can be done as per the requirement and eligibility criterion.

\section{REFERENCES :}

1. Gordan FD, Anastopoulos HT, Crenshaw W et al.(1997),The successful treatment of symptomatic, refractory hepatic hydrothorax with transjugular intrahepatic portosystemic shunt. Hepatology 25,$6 ; 1366-9$.

2. Zenda T, Miyamoto S, Murata S et al .(1998),Detection of diaphragmatic defect as the cause of severe hepatic hydrothorax with magnetic resonance imaging .The American Journal of Gastroenterology ,93,11;2288.

3. Lin CC,Wu JC,Chang SC et al.(2000), Resolution of refractory hepatic hydrothorax after chemical pleurodesis with minocycline. Zhonghua Yi Xue Za Zhi, 63;704-9.

4. Ikard RW and Sawyers JL.(1980),Persistent hepatic hydrothorax after peritoneo-jugular shunt. Archives of Surgery, 115;1125-7.

5. John S, Paul M and Murthy U.(2009),An unusual presentation of cirrhotic pleural effusion in a patient with no ascites: a case report. Cases Journal, 2;6767. 